\title{
A NOTE ON THE STRUCTURE OF MOORE GROUPS
}

\author{
BY LEWIS C. ROBERTSON
}

Communicated by George D. Mostow, September 23, 1968

1. Introduction. A locally compact group $G$ will be called a Moore group if every continuous irreducible unitary representation of $G$ is finite dimensional. Let [Moore] denote the class of all Moore groups, and let $[Z]$ denote the class of all locally compact groups such that $G / Z(G)$ is a compact group, where $Z(G)$ denotes the center of $G$. $S$. Grosser and M. Moskowitz introduced the classes [Moore] and $[Z]$, and made considerable progress on unifying and organizing the study of various "compactness conditions" in locally compact groups. (See [2], [3], and [4].) Grosser and Moskowitz have shown that $[Z] \subset[$ Moore], [3, Theorem 2.1, p. 369], and C. C. Moore has recently shown that $G \in$ [Moore] implies that $G$ is an inverse limit of finite extensions of groups $H_{\alpha} \in[Z]$ (see Theorem 3A below). Other results on Moore groups are obtained below by introducing the notion of Takahashi groups. Let [Tak] denote the class of all locally compact groups $G$ such that the derived group $G^{\prime}$ has compact closure, and $G$ is maximally almost periodic, i.e., there exists a monomorphism from $G$ into a compact group. The main results can be stated as follows:

Theorem 1. A group $G$ satisfies $G \in$ [Moore] if and only if $G$ contains a characteristic subgroup $H$ such that $H$ has finite index in $G$ and $H \in[$ Tak $]$.

THEOREM 2. $A$ group $G$ satisfies $G \in$ [Moore] if and only if $G$ is a semidirect product $G=R^{n} \times_{\phi} B$, where $B \in$ [Moore] has a compact identity component $B_{e}$, and $B$ contains a normal subgroup $H$ with finite index such that $R^{n} \times_{\phi} H$ is a direct product $R^{n} \times H$.

Theorem 2 may be interpreted as a type of generalized Freudenthal-Weil theorem (see Theorem 3C below). Consequences of Theorem 1 are that quotient groups of Takahashi groups are Takahashi groups, and (closed) subgroups of Moore groups are Moore groups. (This behavior is a pleasant contrast to results such as the following:

(1) Closed subgroups of [Z]-groups need not be [Z]-groups.

(2) $G / H$ need not be in [MAP] even when $G \in[\mathrm{MAP}]$ and $H$ is a closed characteristic subgroup of $G$.)

It follows that the class [Moore] is stable under subgroups, quotient groups, inverse limits, and finite extensions; hence the class 
[Moore] constitutes a very well-behaved common generalization of compact groups and abelian groups. (See Theorem 3A for the appropriate notion of inverse limit.) Theorem 1 also implies that every Takahashi group is a Moore group, and hence rounds out the Takahashi duality theorem [9] by showing that the dual structure is based on the set of all equivalence classes of irreducible unitary representations. The inclusion [Tak] $\subset$ [Moore] helps to clarify the relationships between a large number of "compactness" conditions which are summarized in $\$ 5$.

2. Definitions and notation. Notation and terminology are taken from Grosser and Moskowitz for those groups which are discussed in [2], [3], and [4]. Let [MAP] denote the class of locally compact groups which are maximally almost periodic. The classes [MAP], [Moore], [Tak] and [Z] are discussed in the introduction. We will also use the following classes:

[Kur] = Kuranishi groups=locally compact $G$ such that $G \in$ [MAP], and $G / G_{e}$ is compact, where $G_{e}$ denotes the identity component of $G$.

$[\mathrm{FC}]^{-}=$class of locally compact $G$ such that every conjugacy class has compact closure.

A list of definitions of a large number of related properties is available in \$5. (See [4] for a more detailed discussion.)

\section{Background material.}

Theorem 3A (Characterization of Moore Groups). A group $G$ satisfies $G \in$ [Moore] if and only if there is a family $\left\{K_{\alpha}\right\}$ of compact normal subgroups of $G$ such that $\cap K_{\alpha}=e$, and each $G_{\alpha}=G / K_{\alpha}$ is a finite extension of a group $H_{\alpha} \in[Z]$. In particular, the class [Moore] is stable under finite extensions.

Proof. This is as yet an unpublished result of C. C. Moore. Use is made of a nonseparable version of Thoma's Theorem [10].

Theorem 3B (Characterization of Kuranishi Groups). $A$ group $G$ satisfies $G \in$ [Kur] if and only if $G$ is a semidirect product $\mathrm{G}=R^{n} X_{\phi} K$, where $R^{n}$ is a vector group, and $K$ is a compact group which contains a subgroup $H$ such that $H$ has finite index in $K$, and $R^{n} \times_{\phi} H$ is a direct product $R^{n} \times H$.

Proof. The structure theorem for the most general $G \in[$ Kur $]$ was obtained by Murakami [8, Theorem 1, p. 120]. (See also [6, Corollary XII.I, p. 56], and [7, Lemma 2, p. 41]). Murakami develops a 
nice application to the study groups with equal left and right uniformities.

Theorem 3C (FReUdenthal-WeIL). A connected group $G$ satisfies $G \in$ [MAP] if and only if $G=R^{n} \times K$, the direct product of a vector group and a compact group.

Proof [1, Theorem 16.4.6, p. 303].

Theorem 3D (Structure Theorem for Groups $G \in[\mathrm{FC}]^{-}$). $G$ satisfies $G \in[\mathrm{FC}]-$ if and only if there is a compact normal subgroup $K$ such that $G$ is an extension $e \rightarrow K \rightarrow G \rightarrow V \times D \rightarrow e$, where $V$ is a vector group, and $D \in[\mathrm{FC}]-$ is discrete. It follows that every $G \in[\mathrm{Tak}]$ satisfies $G=R^{n} \times H$, where the identrty component $H_{0}$ is compact.

Proof. To appear. The proof uses [4, Corollary 3.22, p. 50].

Theorem 3E (Stability Theorems). Various results show that an appropriate group $G$ contains an n-dimensional normal vector group if $G$ contains a normal subgroup of the form $R^{n} \times H$. See [2, Lemma $1, p$. 328], [6, Theorem X, p. 34], and [4, Theorem 1.1]. This existence of stable vector subgroups also applies to many situations where $G$ acts as a group of automorphisms of $R^{n} \times H$, rather than just action by restriction of inner automorphisms.

\section{Proof of Theorems 1 and 2.}

Proof of Theorem 1. To establish that [Tak] $\subset$ [Moore], start by assuming that $G$ is discrete, and then move on to the case where $G$ is a Lie group with an abelian identity component. The case where $G$ is a Lie group can then be handled by studying the restriction of inner automorphisms to the compact semisimple group $\left(G_{\odot}\right)^{\prime}$. (Use $[5, \S 6$ and Corollary 6.5, p. 122].) Every $G \in[$ Tak] satisfies $G \in[$ SIN]. and hence there are arbitrarily small compact normal subgroups with Lie group quotients. Conversely, if $G \in$ [Moore], then use Theorem $3 A$ and the inclusion $[Z] \subset[F D]-$. (See definition 5.2, and also [2, Corollary 1, p. 331].) The subgroup $H$ can be chosen as the union of all conjugacy classes which have compact closure.

Proof of Theorem 2. Use Theorems $1,3 \mathrm{D}$ and $3 \mathrm{E}$ to obtain a normal subgroup $R^{n} \times M$ of finite index, where $M \in$ [Tak], and $R^{n}$ is normal in $G$. The subgroup $P$ of (topologically) periodic elements of $R^{n} \times M$ must satisfy $P \subset M$, and $P$ is closed by [4, Theorem 3.16]. Moreover, [4, Theorem 3.16] shows that $G / P$ is a finite extension of a torsion free abelian group $A=R^{n} \times D$ where the dual group $D$ is compact connected. Apply Theorem $3 \mathrm{E}$ to the action of $G$ on the 
dual group $\left(R^{n}\right)^{\wedge} \times D^{\wedge}$, and thus obtain an isomorphic copy $D_{1}$ of $D$ such that $A=R^{n} \times D_{1}$ with both fartors stable under $G$. Let $H$ be the inverse image in $G$ of $D_{1}$, and then define $B$ by applying Theorem 3B to the Kuranishi group $G / H$.

\section{Definitions and relationships.}

5.1. $[\mathrm{FC}]^{-}=$class of locally compact groups $G$ such that every conjugacy class of $G$ has compact closure.

5.2. $[\mathrm{FD}]^{-}=$class of locally compact groups $G$ such that the derived group $G^{\prime}$ has compact closure.

5.3. [FIA]- = class of locally compact groups $G$ such that the group of inner automorphisms has compact closure in the group Aut $[G]$ of all homeomorphic automorphisms.

5.4. $[\mathrm{IN}]=$ class of locally compact groups $G$ such that the identity $e \in G$ is contained in some compact neighborhood which is invariant under all inner automorphisms of $G$. (This is called the invariant neighborhood property.)

5.5. $[\mathrm{Kur}]=$ Kuranishi groups = locally compact $[\mathrm{MAP}]$ groups such that $G / G_{e}$ is compact, where $G_{\theta}$ denotes the identity component of $G$.

5.6. $[\mathrm{MAP}]=$ maximally almost periodic groups = locally compact $G$ such that there exists a monomorphism from $G$ into some compact group.

5.7. $[$ Moore] $=$ Moore groups $=$ locally compact $G$ such that every continuous irreducible unitary representation of $G$ is finite dimensional.

5.8. $[$ Mur $]=$ Murakami groups $=[\mathrm{MAP}] \cap[\mathrm{SIN}]$.

5.9. $\left[\mathrm{P}_{1}\right]=$ class of all discrete groups.

5.10. $\left[\mathrm{P}_{2}\right]=$ class of locally compact $G$ such that the center $Z(G)$ contains a vector group $V=R^{n}$ such that $G / V \in\left[\mathrm{P}_{1}\right]$, that is the identity component $G_{e}$ is an open vector group (perhaps trivial), and $G_{e} \subset Z(G)$.

5.11. $\left[\mathrm{P}_{3}\right]=$ class of locally compact $G$ such that there exists a compact normal $K$ with $G / K \in\left[\mathrm{P}_{2}\right]$.

5.12. $\left[\mathrm{P}_{4}\right]=$ class of locally compact $G$ such that there exists a characteristic subgroup $H$ of finite index in $G$ such that $H \in\left[\mathrm{P}_{\mathbf{8}}\right]$.

5.13. $\left[\mathrm{P}_{5}\right]=$ class of all locally compact $G$ such that there exists an open normal $H$ with $H \in\left[\mathrm{P}_{\mathbf{z}}\right]$. 
5.14. $[\mathrm{SIN}]=$ subclass of [IN] consisting of those $G$ such that every neighborhood of $e$ contains an invariant neighborhood of $e$. (This is called the small invariant neighborhoods property.)

5.15. $[\mathrm{Tak}]=$ Takahashi groups $=[\mathrm{MAP} \cap[\mathrm{FD}]-$.

5.16. $[\mathrm{UM}]=$ class of locally compact unimodular groups.

5.17. $[Z]=$ class of locally compact $G$ such that $G / Z(G)$ is compact, where $Z(G)$ denotes the center of $G$.

5.18. Relationships. The table below displays relationships between the various properties. (Many of the relationships indicated below are theorems from [2] and [4].) Here an asterisk is to be interpreted as blocking an implication arrow, otherwise for properties $X$ and $Y$ which are horizontally or vertically adjacent, the interpretation is $X \Rightarrow Y$ if $Y$ is either to the right of $X$ or below $X$. (For instance, $[\mathrm{Tak}] \Rightarrow[\mathrm{FD}]-$ $\Rightarrow[\mathrm{FC}]^{-} \Rightarrow\left[\mathrm{P}_{3}\right] \Rightarrow\left[\mathrm{P}_{4}\right] \Rightarrow[\mathrm{IN}] \Rightarrow\left[\mathrm{P}_{5}\right]$ is a typical string of implications.) An implication of the form $[\mathrm{X}] \Rightarrow\left[P_{j}\right]$ may be regarded as a structure theorem for groups $G \in[\mathrm{X}]$.

\begin{tabular}{|c|c|c|c|c|c|}
\hline & & [Tak] & [FIA]- & [SIN & \\
\hline & & {$[\mathrm{FD}]^{-}$} & {$[\mathrm{FC}]^{-}$} & [IN] & \\
\hline & [Kur] & & {$\left[\mathrm{P}_{3}\right]$} & & [MA \\
\hline Z] [Tak] & [Moore] & [Mur] & {$\left[\mathrm{P}_{4}\right]$} & [IN] & [UM] \\
\hline & & [MAP] & & {$\left[\mathrm{P}_{5}\right]$} & \\
\hline
\end{tabular}

BIBLIOGRAPHY 1964.

1. J. Dixmier, Les $C^{*}$-algèbras et leurs représentations, Gauthier-Villars, Paris,

2. S. Grosser and M. Moskowitz, On central topological groups, Trans. Amer. Math. Soc. 127 (1967), 317-340.

3. - Representation theory of central topological groups, Trans. Amer. Math. Soc. 129 (1967), 361-390.

4. Compactness conditions in topological groups. I, II, J. Reine Agnew. Math. (to appear).

5. S. Helgason, Differential geometry and symmetric spaces, Academic Press, New York, 1962.

6. K. H. Hofmann and P. Mostert, Splitting in topological groups, Mem. Amer. Math. Soc. No. 43, (1963).

7. M. Kuranishi, On non-connected maximally almost periodic groups, Tôhoku Math. J. 2 (1950), 40-46.

8. S. Murakami, Remarks on the structure of maximally almost periodic groups, Osaka Math. J. 2 (1950), 119-129. 
9. S. Takahashi, $A$ duality theorem for representable locally compact groups with compact commutator subgroup, Tôhoku Math. J. 2 (1952), 115-121.

10. E. Thoma, Über unitäre Darstellungen abzählbar, diskreter Gruppen, Math. Ann. 153 (1964), 111-138.

University of Washington, Seattle, Washington 98105

\section{TWO SIDED IDEALS OF OPERATORS}

\section{BY HORACIO PORTA}

Communicated by Paul Halmos, September 30, 1968

1. Let $X$ be a Banach space, and $B(X)$ the Banach algebra of all bounded linear operators in $X$. The closed two sided ideals of $B(X)$ (actually, of any Banach algebra) form a complete lattice $L(X)$. Aside from very concrete cases, $L(X)$ has not yet been determined; for instance, when $X=l^{p}, 1 \leqq p<\infty, L(X)$ is a chain (i.e., totally ordered) with three elements: $\{0\}, B(X)$ and the ideal $C(X)$ of compact operators (see [3]). On the other hand, it is known $[2,5.23]$ that for $X=L^{p}, 1<p<\infty$, the lattice $L(X)$ is not a chain. A treatment for $X$ a Hilbert space of arbitrary dimension can be found in [4]. We aim to exhibit here a Banach space $X$ such that $L(X)$ is both "long" and "wide." Precisely, we have

Proposition. There exists a real Banach space $X$ with the properties:

(i) $X$ is separable, isometric to its dual $X^{*}$, and reflexive;

(ii) it is possible to assign a closed two sided ideal a (F) $\subset B(X)$ to each finite set of positive integers $\mathcal{F}$, in such a way that the mapping $\mathcal{F} \rightarrow \mathfrak{a}(\mathcal{F})$ is injective and inclusion preserving in both directions: $\Im \subseteq \mathcal{S}$ if and only if $\mathfrak{a}(\mathfrak{F}) \subseteq \mathfrak{a}(\mathcal{G})$.

The example is described below, in $\$ 3$.

2. In the sequel, all Banach spaces are real (the complex case can be dealt with similarly). If $X, Y$ are Banach spaces, $\mathfrak{m}(Y, X)$ denotes the set of operators $T \in B(X)$ that can be factorized through $Y$, i.e., such that $T=S Q$ for suitable bounded linear operators $Q: X \rightarrow Y$, $S: Y \rightarrow X$. If $Y$ is isomorphic (as a Banach space) to its square $Y \times Y$ ( $X$ means cartesian product), then (see [6, Proposition 1.2] or [2, Theorem 5.13]) $\mathfrak{m}(Y, X)$ is a two sided ideal of $B(X) \cdot \mathfrak{a}(Y, X)$ will denote the (uniform) closure of $\mathfrak{m}(Y, X)$; thus, if $Y$ is isomorphic to $Y \times Y, \mathfrak{a}(Y, X)$ is a closed two sided ideal of $B(X)$.

In all that follows, subspace means closed lineal subspace; a sub- 